\title{
Long-Term Effect of Different Organic Nutrient Management Practices on Growth, Yield of Field Bean (Dolichos lablab L.) and Soil Properties
}

\author{
M.Y. Ullasa ${ }^{1}$, S. Pradeep ${ }^{1}$ and A.H. Kumar Naik ${ }^{2 *}$ \\ ${ }^{1}$ Organic Farming Research Centre, UAHS, Shivamogga, Karnataka, India \\ ${ }^{2}$ All India Coordinated Research Project on Groundnut, Hiriyur, Karnataka, India \\ *Corresponding author
}

Keywords

Vermi-compost, FYM, $N$ equivalent, Microbial population

Article Info

Accepted:

04 September 2018

Available Online:

10 October 2018

\section{A B S T R A C T}

A field experiment was conducted during Kharif season of five consecutive years from 2013 to 2017, to study the effect of organic nutrient management practices on growth and yield of Field bean (Dolichos lablab L.) in sandy loam soils of Zonal Agricultural and Horticultural Research Station, Navile, Shivamogga, Karnataka. Among the different nutrient management treatments, application of recommended dose of FYM $\left(7.5 \mathrm{t} \mathrm{ha}^{-1}\right)$ along with 100 percent $\mathrm{N}$ equivalent vermi-compost $\left(2 \mathrm{tha}^{-1}\right)$ has recorded significantly higher Dry pod yield (13.2 $\left.\mathrm{q} \mathrm{ha}^{-1}\right)$ and seed yield $(9.32 \mathrm{q} / \mathrm{ha})$ as compared to rest of the treatments. Similarly higher pods per plant ( 23.1 pods/ plant) yield per plant ( $15.9 \mathrm{~g} / \mathrm{plant})$ also recorded with the same treatment and it was closely followed by the treatment which received recommended dose of FYM $\left(7.5 \mathrm{t} \mathrm{ha}^{-1}\right)$ along with application of 100 percent $\mathrm{N}$ equivalent FYM $\left(5 \mathrm{t} \mathrm{ha}^{-1}\right)$. Maximum soil microbial population at different growth stages were also observed in the treatment which received recommended dose of FYM along with 100 percent $\mathrm{N}$ equivalent vermi-compost. The same treatment recorded built up in available nutrient status as compared to initial levels. Hence, application of 2 ton vermicompost along with 7.5 ton FYM is optimum for obtaining highest yield of field bean under organic farming condition.

\section{Introduction}

Field bean usually known as Dolichos bean, Hyacinth bean or Field bean is one of the most ancient crops among cultivated plants. It is a bushy, semi-erect, perennial herb, showing no tendency to climb. It is mainly cultivated either as a pure crop or mixed with finger millet, groundnut, castor, corn, bajra or sorghum in Asia and Africa. It is a multipurpose crop grown for pulse, vegetable and forage. The crop is grown for its green pods, while dry seeds are used in various vegetable food preparations. It is also grown in home gardens as annual crop or on fences as perennial crop. It is one of the major sources of protein in the diets in southern states of India. Within India, Lablab is a field crop mostly confined to the peninsular region and cultivated to a large extent in Karnataka and adjoining districts of Tamil Nadu, Andhra Pradesh and Maharashtra. Karnataka contributes a major share, accounting for nearly 90 per cent in terms of both area and 
production in the country. Karnataka state records production of about 18,000 tonnes from an area of 85,000 hectares. Outside India, the crop is cultivated in East Africa, with similar uses, and in Australia as a fodder crop.

Lablab is remarkably adaptable to wide areas under diverse climatic conditions such as arid, semi-arid, sub-tropical and humid regions where temperatures vary between $22^{\circ} \mathrm{C}-35^{\circ} \mathrm{C}$, low lands and uplands and many types of soils and the $\mathrm{pH}$ varying from 4.4 to 7.8 . Being a legume, it can fix atmospheric nitrogen to the extent of $170 \mathrm{~kg} / \mathrm{ha}$ besides leaving enough crop residues to enrich the soils with organic matter. It is a drought tolerant crop and grows well in dry lands with limited rainfall. The crop prefers relatively cool seasons (temperature ranging from $14-28^{\circ} \mathrm{C}$ ) with the sowing done in July-August. It starts flowering in short days (11-11.5 hour's day length) and continues indeterminately in spring. Hyacinth bean flowers throughout the growing season. Hence, there is a scope to develop the organic nutrient management practices for field bean under rain fed condition. Continuous use of only chemical fertilizers in intensive cropping system is leads to imbalance of nutrients in soil, which has an adverse effect on soil health and also on sustainable crop yields. Hence, In order to achieve the sustainability in crop production development of Organic package of practices for major crops in general and field bean in particular is the need of the hour. Organic farming is a holistic system designed to optimize the productivity and fitness of diverse communities in the agro-ecosystem including living organisms viz., soil organisms, plants, livestock and human being etc., organic farming plays a vital role in maintaining biological diversity, decrease soil and ground water contamination, optimize biological productivity (Watson et al., 2002), maintain long-term soil fertility by optimizing conditions for biological activity in the soil (Ramesh et al., 2005).

Production technology for organic field bean primarily involves three management practices viz., efficient crop management, appropriate nutrient management and effective plant protection measures. Among them nutrient management plays important role. In addition to organic manures such as FYM, recycling of organic wastes through composting, green manures and biological inputs like vermicompost and bio-fertilizers etc., constitute important components for plant nutrient management in organic farming and it is indispensable to identify the better source of nutrient and quantity to meet the nutrient requirement of field bean. The main objective of the present investigation was to develop organic nutrient management practice for field bean and to study the long term effect of input on soil physical chemical and biological properties of soil.

\section{Materials and Methods}

A field experiment was conducted at Organic Farming Research Centre, Navile, Shivamogga, Karnataka $\left(13^{0} 58^{\prime} 30^{\prime \prime} \mathrm{N}\right.$ and $75^{\circ}$ 34' 37 " E) during Kharif season five consecutive years from 2013 to 2017, to study the Effect of different organic nutrient management practices on growth, yield of Field bean and soil properties. The soil of experimental site was slightly acidic in reaction $\left(\mathrm{p}^{\mathrm{H}}\right.$ 5.89) with normal electrical conductivity $(0.01 \mathrm{dS} / \mathrm{m})$, medium organic carbon (4.02 g/kg), low available nitrogen (211 kg/ha), high available phosphorous (112 $\mathrm{kg} / \mathrm{ha}$ ) and medium available potassium (144 $\mathrm{kg} / \mathrm{ha}$ ). The experiment was laid out in randomized block design with nine treatments and replicated thrice with gross plot size of 5.4 $\mathrm{x} 4.5 \mathrm{~m}$ and in-vitro culture of microbial load in the rhizosphere soil was analyzed in completely randomized design. Recommended 
dose of FYM and nitrogen for Field bean under rain fed situation is 7.5 tonesha $^{-1}$ and 25 $\mathrm{kg} / \mathrm{ha}$, respectively. Experiment consisted of nine different manurial treatments. Particulars of nutrients applied in each treatment are specified in Table 1.

Entire quantity of manures was applied to each plots 15 days before sowing of the crop and incorporated into the soil. Field bean seeds were sown in the main field at a spacing $90 \times 30 \mathrm{~cm}$. Crop received optimum rainfall during entire growing period and lifesaving irrigations was provided at critical stages of the crop. Two inter-cultivation operations and one hand weeding were carried out to suppress the weeds. Neem oil was sprayed to control the pest hazards.

Soil samples were collected from each plot before and after the experiment at $0-30 \mathrm{~cm}$ depth. The soil samples were dried in shade, sieved (2 $\mathrm{mm}$ sieve) and analyzed for $\mathrm{pH}$, organic carbon, available nitrogen, available phosphorus and available potassium content. The $\mathrm{pH}$ (soil: water 1:2.5) was measured with the help of $\mathrm{pH}$ meter (Jackson, 1973); organic carbon by Walkley and Black chromic acid digestion method as described by Jackson (1973). Available nitrogen in soil was determined by using Kel-plus nitrogen distillation unit (Subbaiah and Asija, 1956).

The available phosphorus was determined following the procedure described by Jackson (1973) and potassium was determined flame photometrically by neutral normal ammonium acetate extraction method (Hanway and Heidel, 1952). The pertinent growth, yield, soil nutrient status and soil microbial load parameters were recorded using standard procedures. All the parameters were then analyzed for drawing conclusion using analysis of variance (ANOVA) procedure (Gomez and Gomez 1984).

\section{Results and Discussion}

\section{Effect of organic nutrient management practices on soil biological properties}

The data of soil microbial analysis carried out during each year revealed that the population of micro-organisms was increased significantly from 2013 to 2017, during 2013 the population of bacteria $-20.33 \times 10^{5}$, Fungi$5.67 \times 10^{4}$, Actinomycetes- $3.33 \times 10^{3}$, PSMs$4.33 \times 10^{3}$, N-fixers $8.00 \times 10^{3} \mathrm{CFU} / \mathrm{g}$ soil of the experimental site, Application of organic manures significantly influences the soil biological property.

The maximum soil microbial population such as Bacteria- $60.33 \times 10^{5}, 84.00 \times 10^{5}, 63.00 \times$ $10^{5}$, Fungi- $16.67 \times 10^{4}, 26.00 \times 10^{4}, 15.00 \times$ $10^{4}$, Actinomycetes- $10.00 \times 10^{3}, 16.33 \times 10^{3}$, $11.67 \times 10^{3}$, PSMs-16.00 x $10^{3}, 28.33 \times 10^{3}$, $18.33 \times 10^{3}$, N-fixers 19.33. x $10^{3}, 32.67 \times$ $10^{3}, 21.33 \times 10^{3} \mathrm{CFU} / \mathrm{g}$ soil at $30 \mathrm{DAS}, 60$ DAS and at harvest respectively, was recorded in the treatment applied with Rec. FYM +100 $\% \mathrm{~N}$ equivalent to vermi-compost followed by the treatment $\mathrm{T} 1$.

There is a significant increase in soil microbial population from initial to 60 days there after the microbial population was decreased.

The addition of organic inputs enhanced the microbial counts in soil significantly over the years, which might be due to carbon addition and changes in physico-chemical and biological properties of soil (Table 2).

The vermi-compost contain higher amount of growth promoting substances, vitamins, and enzymes, and increases the root biomass production, which resulted in higher production of root exudates and increasing the beneficial bacteria, fungi and actinomycetes population in rhizosphere region (Upadhyay et al., 2011; Jayanthi et al., 2014). 
Effect of organic nutrient management practices on growth of field bean

Among the different nutrient management practices the application of 100 percent $\mathrm{N}$ equivalent vermin-compost ( 2 tonnes/ ha) along with recommended FYM (7.5 tonnes/ ha) $\left(\mathrm{T}_{5}\right)$ recorded significantly higher plant height at harvest $(64 \mathrm{~cm})$ as compared to rest of the treatments (Table 3). Higher dry matter production in plants results in effective utilization of applied resources and it is also the real indicator of plant growth. In the present investigation significantly higher dry matter production $(31.60 \mathrm{~g} / \mathrm{plant})$ at harvest stage was also recorded in the treatment $\mathrm{T}_{5}$ as compared to rest of the treatments except the treatment $\mathrm{T}_{1}$ (Table 3 ).

Higher dry matter production is the prerequisite for higher yield per plant. The higher growth parameters associated with treatment $\mathrm{T}_{5}$ which received 100 per cent $\mathrm{N}$ equivalent vermi-compost along with recommended dose of FYM is attributed to higher quantity of Nitrogen $\left(55 \mathrm{~kg} \mathrm{ha}^{-1}\right)$ and other nutrients made available to crop through supply of 2 ton vermi-compost along with 7.5 t FYM to the crop. Further cementing action of polysaccharides and other organic compounds released during the decomposition of organic matter supplied through FYM and/or vermi-compost that provided better soil environment thus leading to taller plants, increased number of leaves and branches and in turn the final yield.

The organic manures have slow release of nitrogen due to its slow mineralization which induces the availability of nutrients commensurate with the growth and development of the plants and thus results in higher growth parameters (Channabasanagowda et al., 2008). Better availability of plant growth regulators and humicacid in vermicompost results in the increased activity of microbes (Arancon et al., 2004). Microbes like fungi bacteria, yeasts, actinomycetes, algae, etc. are capable of producing auxins, gibberellins etc. in appreciable quantity during vermicomposting (Arancon et al., 2004) which affect plant growth appreciably (Arancon et al., 2006). These results are in conformity with the findings of Mittollia et al., (2015).

\section{Effect of organic nutrient management practices on yield parameter and yield of field bean}

The yield of the crop is an expression of growth and development of the crop. Among the different treatments the application of 100 percent $\mathrm{N}$ equivalent vermin-compost (2 tonnes/ ha) along with recommended FYM (7.5 tonnes/ ha) recorded significantly higher pods per plant (23.1) as compared to rest of the treatments. However, it was closely followed by treatment which received 100 per cent $\mathrm{N}$ equivalent FYM along with recommended FYM (22.1 pods per plant) and the treatment $\mathrm{T}_{8}$ which received 125 per cent vermi- compost alone. Less number of pods per plant (18.9) was recorded in $\mathrm{T}_{2}$ which received 75 percent $\mathrm{N}$ equivalent $\mathrm{FYM}$.

The per plant grain yield of the Field bean found higher in $\mathrm{T}_{5}(15.9 \mathrm{~g} / \mathrm{plant})$ followed by $\mathrm{T}_{1}(15.1 \mathrm{~g} / \mathrm{plant})$ and both were significantly superior over the rest of the treatments. The lowest grain yield per plant was recorded in $\mathrm{T}_{2}$ (12.5 g/plant) which received 75 percent $\mathrm{N}$ equivalent FYM (Table 3). Improvement in yield attributes could be due to higher quantity of macro and micronutrients added to soil in the form of FYM and vermi- compost (Table 1) resulting in increased availability of nutrients in root zone thus more uptake by crop resulting in higher values of yield attributing characters. These results are inconformity with the result obtained by Mittollia et al., 2015. 
Table.1 Treatment details and quantity of nutrients applied in each treatment

\begin{tabular}{|c|c|c|c|c|}
\hline Tr. No. & Treatments & $\begin{array}{l}\text { Total quantity of } \\
\text { FYM applied } \\
\text { (tonnes/ha) }\end{array}$ & $\begin{array}{c}\text { Total quantity } \\
\text { of vermi- } \\
\text { compost applied } \\
\text { (tones/ha) }\end{array}$ & $\begin{array}{c}\text { Total N } \\
\text { supplied } \\
\text { (kg/ha) }\end{array}$ \\
\hline $\mathbf{T}_{1}$ & $\begin{array}{l}\text { Recommended FYM }\left(7.5 \mathrm{tha}^{-1}\right)+ \\
100 \% \mathrm{~N} \text { equivalent FYM }\end{array}$ & 12.5 & 0 & 62.5 \\
\hline $\mathbf{T}_{2}$ & $75 \% \mathrm{~N}$ equivalent FYM & 3.75 & 0 & 18.25 \\
\hline $\mathbf{T}_{3}$ & $100 \%$ N equivalent FYM & 5 & 0 & 25.00 \\
\hline $\mathbf{T}_{4}$ & $125 \% \mathrm{~N}$ equivalent FYM & 6.25 & 0 & 31.25 \\
\hline $\mathrm{T}_{5}$ & $\begin{array}{l}\text { Recommended FYM }\left(7.5 \mathrm{t} \mathrm{ha}^{-1}\right)+ \\
100 \% \mathrm{~N} \text { equivalent vermicompost }\end{array}$ & 7.5 & 2.0 & 62.5 \\
\hline $\mathrm{T}_{6}$ & $75 \% \mathrm{~N}$ equivalent vermicompost & 0 & 1.5 & 18.75 \\
\hline $\mathrm{T}_{7}$ & $100 \% \mathrm{~N}$ equivalent vermicompost & 0 & 2.0 & 25.00 \\
\hline $\mathrm{T}_{8}$ & $125 \% \mathrm{~N}$ equivalent vermicompost & 0 & 2.5 & 31.25 \\
\hline $\mathrm{T}_{9}$ & $\begin{array}{l}50 \% \mathrm{~N} \text { equivalent } \mathrm{FYM}+50 \% \mathrm{~N} \\
\text { equivalent vermicompost }\end{array}$ & 2.5 & 1.0 & 25.00 \\
\hline
\end{tabular}

Table.3 Growth and yield parameters of field bean as influenced by different nutrient management practices (pooled data of five years)

\begin{tabular}{|c|c|c|c|c|c|}
\hline $\begin{array}{l}\text { Tr. } \\
\text { No. }\end{array}$ & Treatments & $\begin{array}{c}\text { Plant } \\
\text { height } \\
\text { (cm) }\end{array}$ & $\begin{array}{l}\text { Dry matter } \\
\text { (g) }\end{array}$ & $\begin{array}{l}\text { No. of pods } \\
\text { plant }^{-1}\end{array}$ & $\begin{array}{c}\text { yield plant } \\
1(\mathrm{~g})\end{array}$ \\
\hline $\mathbf{T}_{1}$ & $\begin{array}{l}\text { Recommended FYM }\left(7.5 \mathrm{tha}^{-1}\right)+ \\
100 \% \mathrm{~N} \text { equivalent FYM }\end{array}$ & 58.8 & 29.10 & 22.1 & 15.1 \\
\hline $\mathrm{T}_{2}$ & $75 \% \mathrm{~N}$ equivalent FYM & 49.8 & 22.10 & 18.9 & 12.5 \\
\hline$\overline{T_{3}}$ & $100 \% \mathrm{~N}$ equivalent FYM & 50.6 & 23.60 & 19.9 & 12.8 \\
\hline $\mathrm{T}_{4}$ & $125 \% \mathrm{~N}$ equivalent FYM & 53.6 & 25.90 & 20.4 & 13.3 \\
\hline $\mathrm{T}_{5}$ & $\begin{array}{l}\text { Recommended FYM }\left(7.5 \mathrm{t} \mathrm{ha}^{-1}\right)+ \\
100 \% \mathrm{~N} \text { equivalent vermicompost }\end{array}$ & 64.0 & 31.60 & 23.1 & 15.9 \\
\hline$T_{6}$ & $75 \% \mathrm{~N}$ equivalent vermicompost & 51.5 & 23.60 & 19.6 & 12.4 \\
\hline $\mathrm{T}_{7}$ & $100 \% \mathrm{~N}$ equivalent vermicompost & 53.2 & 25.30 & 20.3 & 13.0 \\
\hline $\mathrm{T}_{8}$ & $125 \% \mathrm{~N}$ equivalent vermicompost & 57.5 & 28.20 & 21.5 & 13.9 \\
\hline $\mathrm{T}_{9}$ & $\begin{array}{l}50 \% \mathrm{~N} \text { equivalent } \mathrm{FYM}+50 \% \mathrm{~N} \\
\text { equivalent vermicompost }\end{array}$ & 53.2 & 26.80 & 20.1 & 12.9 \\
\hline & $\begin{array}{lr}\text { S.Em. } \pm \\
\end{array}$ & 4.06 & 4.1 & 0.93 & 0.69 \\
\hline & C.D. at $5 \%$ & 12.19 & 12.2 & 2.78 & 2.08 \\
\hline
\end{tabular}


Table.2 General soil micro flora (CFU g ${ }^{-1}$ of soil) as influenced by nutrient management practices for organic cultivation of field bean

\begin{tabular}{|c|c|c|c|c|c|c|c|c|c|c|c|c|c|c|c|c|c|c|c|c|}
\hline $\begin{array}{c}\text { Micro } \\
\text { flora }\end{array}$ & \multicolumn{4}{|c|}{$\begin{array}{c}\text { Bacteria } \\
\left(\text { CFUs.X 10 }{ }^{5}\right)\end{array}$} & \multicolumn{4}{|c|}{$\begin{array}{c}\text { Fungi } \\
\left(\text { CFUs.X 10 }{ }^{4}\right)\end{array}$} & \multicolumn{4}{|c|}{$\begin{array}{c}\text { Actinomycetes } \\
(\text { CFUs.X 10 } \\
\text { (1) }\end{array}$} & \multicolumn{4}{|c|}{$\begin{array}{c}\text { PSM } \\
\left(\text { CFUs.X 10 }{ }^{3}\right)\end{array}$} & \multicolumn{4}{|c|}{$\begin{array}{c}\text { N-fixers } \\
\left(\text { CFUs.X 10 }{ }^{3}\right)\end{array}$} \\
\hline Initial & \multicolumn{4}{|c|}{20.33} & \multicolumn{4}{|c|}{5.67} & \multicolumn{4}{|c|}{3.33} & \multicolumn{4}{|c|}{4.33} & \multicolumn{4}{|c|}{8.00} \\
\hline $\begin{array}{l}\text { Treatm } \\
\text { ents / } \\
\text { DAS }\end{array}$ & $\begin{array}{c}30 \\
\text { DAS }\end{array}$ & $\begin{array}{c}60 \\
\text { DAS }\end{array}$ & $\begin{array}{c}\text { At } \\
\text { harvest }\end{array}$ & $\begin{array}{c}30 \\
\text { DAS }\end{array}$ & $\begin{array}{c}60 \\
\text { DAS }\end{array}$ & $\begin{array}{c}\text { At } \\
\text { harvest }\end{array}$ & $\begin{array}{c}30 \\
\text { DAS }\end{array}$ & $\begin{array}{c}60 \\
\text { DAS }\end{array}$ & $\begin{array}{c}\text { At } \\
\text { harvest }\end{array}$ & $\begin{array}{c}30 \\
\text { DAS }\end{array}$ & $\begin{array}{c}60 \\
\text { DAS }\end{array}$ & $\begin{array}{c}\text { At } \\
\text { harvest }\end{array}$ & $\begin{array}{c}30 \\
\text { DAS }\end{array}$ & $\begin{array}{c}60 \\
\text { DAS }\end{array}$ & $\begin{array}{c}\text { At } \\
\text { harvest }\end{array}$ & $\begin{array}{c}30 \\
\text { DAS }\end{array}$ & $\begin{array}{c}60 \\
\text { DAS }\end{array}$ & $\begin{array}{c}\text { At } \\
\text { harvest }\end{array}$ & $\begin{array}{c}30 \\
\text { DAS }\end{array}$ & $\begin{array}{c}60 \\
\text { DAS }\end{array}$ \\
\hline $\mathrm{T}_{1}$ & 59.00 & 80.67 & 62.00 & 15.00 & 25.33 & 14.67 & 09.67 & 14.33 & 10.00 & 15.33 & 26.67 & 16.67 & 17.00 & 30.33 & 20.67 & 59.00 & 80.67 & 62.00 & 15.00 & 25.33 \\
\hline $\mathbf{T}_{2}$ & 53.33 & 73.00 & 54.33 & 10.33 & 19.33 & 09.33 & 06.67 & 09.67 & 06.00 & 10.00 & 19.33 & 10.67 & 12.00 & 24.00 & 13.33 & 53.33 & 73.00 & 54.33 & 10.33 & 19.33 \\
\hline $\mathbf{T}_{3}$ & 56.00 & 76.33 & 58.00 & 13.00 & 22.00 & 12.33 & 07.33 & 12.00 & 08.33 & 12.00 & 22.00 & 14.33 & 14.00 & 26.33 & 17.00 & 56.00 & 76.33 & 58.00 & 13.00 & 22.00 \\
\hline $\mathbf{T}_{4}$ & 58.67 & 80.00 & 61.67 & 14.33 & 24.33 & 14.00 & 08.67 & 13.67 & 09.67 & 14.67 & 24.00 & 16.00 & 16.67 & 29.00 & 20.00 & 58.67 & 80.00 & 61.67 & 14.33 & 24.33 \\
\hline$T_{5}$ & 60.33 & 84.00 & 63.00 & 16.67 & 26.00 & 15.00 & 10.00 & 16.33 & 11.67 & 16.00 & 28.33 & 18.33 & 19.33 & 32.67 & 21.33 & 60.33 & 84.00 & 63.00 & 16.67 & 26.00 \\
\hline$T_{6}$ & 54.00 & 74.67 & 56.00 & 11.33 & 20.33 & 11.33 & 07.00 & 10.67 & 07.33 & 10.00 & 20.00 & 12.33 & 13.00 & 24.67 & 15.33 & 54.00 & 74.67 & 56.00 & 11.33 & 20.33 \\
\hline$T_{7}$ & 54.67 & 75.00 & 56.67 & 12.00 & 21.67 & 12.00 & 07.67 & 11.33 & 08.00 & 11.33 & 21.67 & 13.00 & 13.33 & 26.00 & 17.00 & 54.67 & 75.00 & 56.67 & 12.00 & 21.67 \\
\hline$T_{8}$ & 58.00 & 79.33 & 60.00 & 14.00 & 23.00 & 13.33 & 08.33 & 13.67 & 09.00 & 13.00 & 23.33 & 15.33 & 15.33 & 28.67 & 19.00 & 58.00 & 79.33 & 60.00 & 14.00 & 23.00 \\
\hline$T_{9}$ & 56.67 & 79.00 & 58.33 & 13.33 & 22.33 & 13.00 & 08.00 & 12.00 & 08.33 & 12.33 & 22.00 & 15.00 & 14.67 & 27.33 & 18.33 & 56.67 & 79.00 & 58.33 & 13.33 & 22.33 \\
\hline S.Em. \pm & 0.13 & 0.30 & 0.28 & 0.15 & 0.26 & 0.22 & 0.18 & 0.34 & 0.24 & 0.18 & 0.36 & 0.29 & 0.18 & 0.28 & 0.14 & 0.13 & 0.30 & 0.28 & 0.15 & 0.26 \\
\hline $\begin{array}{l}\text { C.D@ } \\
5 \%\end{array}$ & 0.39 & 0.92 & 0.85 & 0.46 & 0.81 & 0.68 & 0.56 & 1.02 & 0.73 & 0.56 & 1.08 & 0.87 & 0.56 & 0.85 & 0.43 & 0.39 & 0.92 & 0.85 & 0.46 & 0.81 \\
\hline
\end{tabular}


Table.4 Pod yield and seed yield of field bean as influenced by different nutrient management practices

\begin{tabular}{|c|c|c|c|c|c|c|c|c|c|c|c|c|c|}
\hline \multirow{2}{*}{$\begin{array}{l}\text { Tr. } \\
\text { No. }\end{array}$} & \multirow[t]{2}{*}{ Treatments } & \multicolumn{2}{|c|}{ 2013-14 } & \multicolumn{2}{|c|}{ 2014-15 } & \multicolumn{2}{|c|}{ 2015-16 } & \multicolumn{2}{|c|}{ 2016-17 } & \multicolumn{2}{|c|}{ 2017-18 } & \multicolumn{2}{|c|}{ Pooled data } \\
\hline & & $\begin{array}{c}\text { Dry } \\
\text { pod } \\
\text { yield } \\
\text { ha }^{-1} \\
(q)\end{array}$ & $\begin{array}{c}\text { Seed } \\
\text { yield } \\
\text { ha }^{-1} \\
(q)\end{array}$ & $\begin{array}{c}\text { Dry } \\
\text { pod } \\
\text { yield } \\
\text { ha }^{-1} \\
(q)\end{array}$ & $\begin{array}{c}\text { Seed } \\
\text { yield } \\
\text { ha }^{-1} \\
(q)\end{array}$ & $\begin{array}{c}\text { Dry } \\
\text { pod } \\
\text { yield } \\
\text { ha }^{-1} \\
(q)\end{array}$ & $\begin{array}{c}\text { Seed } \\
\text { yield } \\
\text { ha }^{-1} \\
(\mathbf{q})\end{array}$ & $\begin{array}{c}\text { Dry } \\
\text { pod } \\
\text { yield } \\
\text { ha }^{-1} \\
(q)\end{array}$ & $\begin{array}{c}\text { Seed } \\
\text { yield } \\
\text { ha }^{-1} \\
\text { (q) }\end{array}$ & $\begin{array}{c}\text { Dry } \\
\text { pod } \\
\text { yield } \\
\text { ha }^{-1} \\
\text { (q) }\end{array}$ & $\begin{array}{c}\text { Seed } \\
\text { yield } \\
\text { ha }^{-1} \\
(q)\end{array}$ & $\begin{array}{l}\text { Dry pod } \\
\text { yield ha } \\
\quad \text { (q) }\end{array}$ & $\begin{array}{c}\text { Seed } \\
\text { yield ha } \\
1 \text { (q) }\end{array}$ \\
\hline T1 & $\begin{array}{l}\mathrm{FYM}+100 \% \mathrm{~N} \\
\text { equivalent FYM }\end{array}$ & 14.78 & 10.74 & 15.64 & 11.10 & 11.12 & 7.23 & 11.48 & 7.69 & 10.48 & 7.02 & 12.70 & 8.75 \\
\hline T2 & $\begin{array}{l}75 \% \mathrm{~N} \text { equivalent } \\
\text { FYM }\end{array}$ & 13.55 & 9.47 & 13.83 & 9.98 & 9.79 & 5.85 & 6.33 & 4.54 & 5.91 & 5.07 & 9.88 & 6.98 \\
\hline T3 & $\begin{array}{l}100 \% \mathrm{~N} \text { equivalent } \\
\text { FYM }\end{array}$ & 13.88 & 9.96 & 14.24 & 10.40 & 10.18 & 6.34 & 7.95 & 5.32 & 7.29 & 5.25 & 10.71 & 7.45 \\
\hline T4 & $\begin{array}{l}125 \% \mathrm{~N} \text { equivalent } \\
\text { FYM }\end{array}$ & 14.36 & 10.45 & 15.50 & 10.93 & 10.91 & 6.87 & 8.96 & 6.09 & 8.07 & 5.89 & 11.56 & 8.04 \\
\hline T5 & $\begin{array}{l}\mathrm{FYM}+100 \% \mathrm{~N} \\
\text { equivalent } \\
\text { vermicompost }\end{array}$ & 14.92 & 10.97 & 15.88 & 11.56 & 11.82 & 7.70 & 12.36 & 8.62 & 11.29 & 7.75 & 13.25 & 9.32 \\
\hline T6 & $\begin{array}{l}75 \% \mathrm{~N} \text { equivalent } \\
\text { vermicompost }\end{array}$ & 13.64 & 9.58 & 14.32 & 9.78 & 10.09 & 5.90 & 8.57 & 5.65 & 7.96 & 5.35 & 10.91 & 7.25 \\
\hline T7 & $\begin{array}{l}100 \% \mathrm{~N} \text { equivalent } \\
\text { vermicompost }\end{array}$ & 14.23 & 10.28 & 15.12 & 10.82 & 10.66 & 6.67 & 9.02 & 5.68 & 8.21 & 5.48 & 11.45 & 7.78 \\
\hline T8 & $\begin{array}{l}125 \% \mathrm{~N} \text { equivalent } \\
\text { vermicompost }\end{array}$ & 14.53 & 10.56 & 15.50 & 11.02 & 10.91 & 7.03 & 9.75 & 6.33 & 9.50 & 6.60 & 12.03 & 8.30 \\
\hline T9 & $\begin{array}{l}50 \% \mathrm{~N} \text { equivalent } \\
\text { FYM }+50 \% \mathrm{~N} \\
\text { equivalent } \\
\text { vermicompost }\end{array}$ & 14.12 & 10.20 & 14.86 & 10.81 & 10.62 & 6.70 & 9.40 & 5.83 & 8.90 & 6.05 & 11.58 & 7.91 \\
\hline & S.Em. \pm & 0.193 & 0.187 & 10.20 & 0.189 & 0.15 & 0.11 & 0.50 & 0.46 & 1.06 & 1.07 & 0.32 & 0.20 \\
\hline & C.D. at $5 \%$ & 0.58 & 0.54 & 0.61 & 0.56 & 0.44 & 0.32 & 1.49 & 1.37 & 7.09 & 10.18 & 0.94 & 0.60 \\
\hline
\end{tabular}


Table.5 Nutrient status of soil as influenced by different nutrient management practices

\begin{tabular}{|c|c|c|c|c|c|c|c|}
\hline Tr No. & Treatments details & $\begin{array}{c}\mathrm{pH} \\
(1: 2.5)\end{array}$ & $\begin{array}{c}\mathrm{EC} \\
\left(\mathrm{dSm}^{-1}\right)\end{array}$ & $\begin{array}{c}\mathrm{OC} \\
(\mathrm{g} / \mathrm{kg})\end{array}$ & $\begin{array}{c}\mathbf{N} \\
(\mathrm{kg} / \mathrm{ha})\end{array}$ & $\begin{array}{c}\mathrm{P}_{2} \mathrm{O}_{5} \\
\text { (kg/ha) }\end{array}$ & $\begin{array}{c}\mathrm{K}_{2} \mathrm{O} \\
\text { (kg/ha) }\end{array}$ \\
\hline T1 & FYM + $100 \% \mathrm{~N}$ equivalent FYM & 5.90 & 0.04 & 4.31 & 262 & 159 & 175 \\
\hline T2 & $75 \% \mathrm{~N}$ equivalent FYM & 5.88 & 0.03 & 4.18 & 210 & 133 & 158 \\
\hline T3 & $100 \% \mathrm{~N}$ equivalent FYM & 5.97 & 0.04 & 4.37 & 232 & 148 & 167 \\
\hline T4 & $125 \% \mathrm{~N}$ equivalent FYM & 6.21 & 0.03 & 4.33 & 247 & 176 & 174 \\
\hline T5 & $\mathrm{FYM}+100 \% \mathrm{~N}$ equivalent vermicompost & 5.80 & 0.03 & 4.33 & 244 & 159 & 185 \\
\hline T6 & $75 \% \mathrm{~N}$ equivalent vermicompost & 5.85 & 0.03 & 3.57 & 201 & 126 & 159 \\
\hline T7 & $100 \% \mathrm{~N}$ equivalent vermicompost & 6.17 & 0.03 & 4.24 & 217 & 143 & 162 \\
\hline T8 & $125 \% \mathrm{~N}$ equivalent vermicompost & 5.75 & 0.03 & 4.24 & 240 & 147 & 158 \\
\hline \multirow[t]{4}{*}{ T9 } & $\begin{array}{l}50 \% \mathrm{~N} \text { equivalent } \mathrm{FYM}+50 \% \mathrm{~N} \\
\text { equivalent vermicompost }\end{array}$ & 6.22 & 0.03 & 4.06 & 222 & 151 & 170 \\
\hline & Initial & 5.89 & 0.01 & 4.02 & 211 & 112 & 144 \\
\hline & $\mathrm{SEm} \pm$ & 0.21 & 0.00 & 0.19 & 11.02 & 7.82 & 11.66 \\
\hline & CD@5(\%) & NS & NS & NS & 32.72 & 23.22 & NS \\
\hline
\end{tabular}


Due to higher yield attributes associated with treatment $\mathrm{T}_{5}$, the same treatment has recorded significantly higher dry pod yield (13.2 q/ha) and seed yield (9.32 q/ha) as compared to rest of the treatments and closely followed by the treatment which received 100 per cent $\mathrm{N}$ equivalent FYM along with recommended FYM $T_{1}$ Table 4. Significantly, higher grain yield associated with treatment $T_{5}$ is attributed to more effective translocation of photosynthates from source to sink (Raja rajan and Sabrinathan, 2006). Application of FYM improves soil physical condition sand NPK content of soil there by increases yield and yield attributes. Rana et al., (2006) and Vyas et al., (2006) also reported favorable effect of FYM on seed yield of rajmash and pigeon pea respectively.

Although the quantity of nutrients supplied both in $\mathrm{T}_{1}$ and $\mathrm{T}_{5}$ are same the yield was highest in $\mathrm{T}_{5}$. This is attributed in treatment $\mathrm{T}_{5} 100$ per cent $\mathrm{N}$ equivalent $\mathrm{N}$ was supplied through vermi- compost. The vermi-compost contains plant growth regulating materials such as humic acids (Atiyeh et al., 2002) and plant growth regulators like auxins, gibberellins and cytokinins (Tomati et al., 1988) which are responsible for increased plant growth and yield of many crops (Atiyeh et al., 2002). These plant growth regulating materials are produced by action of microbes like fungi, bacteria, actinomycetes and earthworms (Edwards 1998). The highest population of soil micro flora at all growth stages was recorded in $\mathrm{T}_{5}$. Vermi-compost provides large particulate surface areas that provide many micro-sites for microbial activities and for strong retention of nutrients (Shi-wei and Fu-zhen 1991). As a result most nutrients are in available form such as nitrates, phosphates, exchangeable calcium and soluble potassium (Edwards 1998). Vermi-compost application also suppresses the growth of many fungi like Pythium, Rhizoctonia and Verticillium; as a result many plant diseases are suppressed when vermicompost is applied in ample quantity in the field (Hoitink and Fahy 1986).Due to all these reasons Treatment $T_{5}$ has recorded highest yield.

\section{Soil nutrient status}

Nutrient status of the soil is influenced by soil physico-chemical and biological properties. Organic manure application will improve the soil fertility and availability of nutrients through slow mineralization and slow release of nutrients which in turn results in availability of nutrients throughout the growing period of the crop (Dudhat et al., 1997). Different nutrient management practices failed to influence the soil $\mathrm{p}^{\mathrm{H}}, \mathrm{EC}$, $\mathrm{OC}$ and $\mathrm{K}_{2} \mathrm{O}$ status significantly. Soil organic carbon is one of the major components of soil organic matter. It is extremely important in all soil processes viz., nutrient availability, soil structure and soil biological activities. The OC content was improved over the years as compared to initial levels $(4.02 \mathrm{~g} / \mathrm{kg})$. Significantly higher organic carbon content in the soil was recorded in $\mathrm{T}_{5}(4.33 \mathrm{~g} / \mathrm{kg})$ as compared to $T_{2}, T_{4}$ and $T_{9}$ and lower organic carbon $\left(4.06 \mathrm{~g} / \mathrm{kg}\right.$ ) was recorded in $\mathrm{T}_{2}$ (Table $5)$.

Soil nitrogen availability depends on $\mathrm{N}$ mineralization and which in turn results in increased root biomass this leads to increased uptake of the nutrients by the plants. In the present investigation, after harvest of the crop the soil nitrogen status found significantly higher in the $\mathrm{T}_{1}\left(262 \mathrm{~kg} \mathrm{ha}^{-1}\right)$ which received recommended dose of FYM (7.5 tonnes/ha) and 100 percent $\mathrm{N}$ equivalent FYM over the rest of the treatment. As compared to initial status (211 kg ha ${ }^{-1}$ ) gradual buildup of Nitrogen content noticed in the treatment $T_{1}$ $\left(262 \mathrm{~kg} \mathrm{ha}^{-1}\right)$. However, the available nitrogen content was low and it was increased slightly with implementation of organic manure 
treatments as compared to initial level. It might be due to organic matter decomposition and slow release of nutrients to the soil (Srinivasulu et al., 2000). The available phosphorus was found significantly higher in $\mathrm{T}_{1}(159 \mathrm{~kg} / \mathrm{ha})$ gradual increase in available $\mathrm{P}$ content was observed as compared to initial values $\left(112 \mathrm{~kg} \mathrm{ha}^{-1}\right)$. This is attributed to release of organic acid during microbial decomposition of organic matter which might encompass in the solubility of native phosphates thus increasing available phosphorus pool in the soil.

In addition the organic anions compete with phosphate ions for the binding sites on the soil particles (Patra et al., 2011). Different nutrient management treatments failed to influence the potassium status of soil significantly. However, the available potassium was found highest in $\mathrm{T}_{5}$ over rest of the treatments (Table 5). Potassium content of soil was also improved over the years as compared to initial level $\left(144 \mathrm{~kg} \mathrm{ha}^{-1}\right)$. These results are in close conformity with the findings of Nagar et al., 2015.

Amongst the different organic nutrient management practices tested for five consecutive years the application of recommended dose of FYM (7.5 tonnes) along with 100 percent $\mathrm{N}$ equivalent vermicompost (2 tonnes/ha) was found superior with respect to growth, development and yield of the field bean.

The gradual improvement in soil physical, chemical and biological properties was noticed over the years. The higher soil micro flora was also recorded with the application of recommended dose of FYM (7.5 tonnes) along with 100 percent $\mathrm{N}$ equivalent vermicompost $\left(2 \mathrm{tha}^{-1}\right)$. Hence, application of 2 ton vermi-compost along with 7.5 ton FYM is optimum for obtaining highest yield of field bean under organic farming condition.

\section{References}

Arancon, N.Q., Edwards, C. A. and Bierman, P. 2006. Influences of vermi-composts on field strawberries: Part 2. Effects on soil microbial and chemical properties. Bioresource Technology. 97: 831-840.

Arancon, N.Q., Edwards, C.A., Bierman, P., Welch, C. and Metzer, J.D. 2004. Influence of vermi-composts on field strawberries: 1. Effect on growth and yields. Bioresource Technology.93: 145-153.

Atiyeh, R.M., Lee, S.S., Edwards, C.A., Arancon, N.Q. and Metzger, J., 2002. The influence of humic acid derived from earthworm-processed organic wastes on plant growth. Bioresource Technology. 84: 7-14.

Badiane, N. N. Y., Chotte, J. L., Pate, E., Masse, D. and Rouland, C., 2001. Use of soil enzyme activities to monitor soil quality in natural and improved fallows in semi arid tropical regions. J.Appl Soil Ecol. 18: 229-238.

Burns, R. G. 1982. Enzyme activity in soil location and a possible role in microbial activity, J. Soil Biol. Biochem. 14: 423427.

Channabasanagowda, Biradar, N.K., Patil, B.N., Awaknavar, J.S., Ninganur, B.T. and Hunge, R. 2008. Effect of organic manures on growth, seed yield and quality of wheat. Karnataka Journal of Agricultural Sciences. 21(3): 366-368.

Dudhat, M. S., Mathukia, K. and Khampara, V. B. 1997. Effect of nutrient management through organic and inorganic source on growth, yield, quality and nutrient uptake by wheat (Triticum aestivum). Indian J. Agron.42 (3): 455-458.

Edwards, C.A. 1998. The use of earthworm in the breakdown and management of organic wastes. In: Earthworm ecology 
(CA Edwards ed), CRC Press, Boca Raton, FL, pp 327-354.

Frankenberger, W. T. and Dick, W. A. 1983. Relationships between enzyme activities and microbial growth and activity indices in soil. J. Soil Sci., 47: 945-951.

Gomez, K. A. and Gomez, A. 1984. Statistical Procedures for Agric. Res. 2nd Ed. John Wiley \& Sons, New York.

Hanway, J.J. and Heidel, H. 1952. Soil analysis methods used in Iowa State College, Soil Testing Lab, Lowa Agronomy, 57: 1-31.

Hoitink, H.A. and Fahy, P. 1986. Basis for the control of soil borne plant pathogen with composts. Annual Review of Phytopathology. 24: 93-114.

Jackson, M.L. 1973. Soil Chemical Analysis. Prentice Hall of India Pvt. Ltd., New Delhi, p. 498.

Jayanthi, L. J., Sekara, B. S., Ameer and Parthasarathi, K., 2014. Influence of vermi-fertilizer on soil quality, yield and quality of chilli (Capsicum annuum L.). Int. Interdisciplinary Res. J., 4(4): 2249-9598.

Mittolia, V. K., Singh, R. K. and Sanjeev Verma. 2015. Performance of Integrated Nutrient Management in Pigeonpea (Cajanus Cajana). Journal of Community Mobilization and Sustainable Development.10 (1): 05-08.

Nagar, R. K., Goud, V. V., Rajesh Kumar and Ravindra Kumar. 2015. Effect of incorporation of FYM, pigeon pea stalk phosphor compost on growth, yield and nutrient uptake in pigeon pea based intercropping system. The Bioscan. 10 (3): 339-343.

Patra, P.S., Sinha, A.C. and Mahesh, S.S., 2011. Yield, nutrient uptake and quality of groundnut (Arachis hypogaea) kernels as affected by organic sources of nutrient. Indian Journal of Argonomy. 56 (3): 237-241.
Raja Rajan, and Sabarinathan, R. 2006. Effect of in situ Moisture Conservation and Nutrient Management Practices on Soil Properties and Yield of Redgram in a Rainfed Alfisol.In: The 18th World Congress of Soil Science.

Rajesh Kumar and Paslawar, A. N. 2017. Effect of conservation tillage on biomass partitioning and quality of pigeon pea based intercropping system under Vidarbha region. The Bioscan. 12 (1): 571-574.

Ramesh, P., Singh, M. and Subba Rao, A., 2005, Organic farming: its relevance to the Indian context. Current Science 88 (4): 561-568.

Rana, M.C., Datt N. and Singh. M., 2006. Effect of Rhizobium in combination with organic and chemical fertilizers on rajmash under dry temperate condition of Himanchal Pradesh. Indian Journal of Agricultural Sciences.76 (3): 151153.

Shi-wei, Z. and Fu-zhen, H., 1991. The nitrogen uptake efficiency from $\mathrm{N}$ labeled chemical fertilizer in the presence of earthworm manure (cast). In: Advances in management and conservation of soil fauna (GK Veeresh, D Rajagopal and CA Viraktamath eds), Oxford and IBH Publishing Co Pvt Ltd, New Delhi, India, pp 539-542.

Srinivasulu, K., Singh, R.P. and Madhavi, K., 2000, Performance of rain fed pigeon pea-based intercropping systems under varying plantings. Crop Research. 20: $56-61$.

Subbaiah, B. Y. and Asija, G. L., 1956. A rapid procedure for the estimation of available nitrogen in soils. Curr. Sci., 25: 259-260.

Tomati, U., Grappelli, A and Galli, E., 1988. The hormone-like effect of earthworm casts on plant growth. Biology and Fertility of Soils. 5(4): 288-294. 
Upadhyay VB, Jain V, Vishwakarma SK, Kumar A. K., 2011. Production potential, soil health water productivity and economic of rice (Oryza sativa) based cropping system under different nutrients sources. Indian J. Agron. 56(4):311-316.

Vepsalainen, M., Kukkonen, S., Vestberg, M., Sirvio, H. and Niemi, R. M., 2001. Application of soil enzyme activity test kit in a field experiment. Soil Biol. Biochem. 33: 1665-1672.
Vyas, A.K., Billore, S. D. and Joshi, O. P. 2006. Productivity and economics of integrated nutrient management in soybean plus pigeon pea intercropping system. Indian Journal of Agricultural Sciences, 76(1): 7-10.

Watson, C.A., Atkinson, D., Gosling, P., Jackson, L.R. and Rayns, F. W., 2002. Managing soil fertility in organic farming systems. Soil Use and Management 18: 239-247.

\section{How to cite this article:}

Ullasa, M.Y., S. Pradeep and Kumar Naik, A.H. 2018. Long-Term Effect of Different Organic Nutrient Management Practices on Growth, Yield of Field Bean (Dolichos lablab L.) and Soil Properties. Int.J.Curr.Microbiol.App.Sci. 7(10): 51-62.

doi: https://doi.org/10.20546/ijcmas.2018.710.007 